\title{
Smarandache Curves of Spacelike Anti-Salkowski Curve with a Timelike Principal Normal According to Frenet Frame
}

\author{
Süleyman ŞENYURT ${ }^{1 *}$ (D), Kemal EREN ${ }^{2}$ (D) \\ ${ }^{1}$ Faculty of Arts and Sciences, Department of Mathematics, Ordu University, Ordu, TURKEY \\ ${ }^{2}$ Faculty of Arts and Sciences, Department of Mathematics, Sakarya University, Sakarya, TURKEY
}

Geliş / Received: 17/09/2019, Kabul / Accepted: 10/07/2020

\begin{abstract}
In this study, we investigate the regular Smarandache curves obtained from the Frenet vectors of the spacelike anti-Salkowski curve with a timelike principal normal. Firstly, we define Smarandache curves depending upon the anti-Salkowski curve. Later, the curvature, torsion and the Frenet vectors of Smarandache curves are determined. Finally, the Frenet apparatuses of the obtained Smarandache curves are expressed as Frenet vectors of the spacelike anti-Salkowski curve. We draw graphic of the obtained Smarandache curves and some related results are given.
\end{abstract}

Keywords: Minkowski space, spacelike anti-Salkowski curve, Smarandache curve, Frenet frame.

\section{Frenet Çatısına Göre Timelike Normalli Spacelike Anti-Salkowski Eğrisinden Elde Edilen Smarandache Ĕgrileri}

\section{$\ddot{O} z$}

$\mathrm{Bu}$ çalışmada timelike normalli spacelike anti-Salkowski eğrisinin Frenet vektörlerinden elde edilen regüler Smarandache eğrilerini araştırdık. İlk aşamada anti-Salkowski eğrisine bağlı Smarandache eğrilerini tanımladık. Daha sonra her bir Smarandache eğrisinin Frenet vektörleri, eğrilik ve torsiyonu hesaplandı. Son olarak elde edilen Smarandache eğrilerin Frenet elemanları spacelike anti-Salkowski eğrisinin Frenet vektörleri cinsinden ifade edildi. Elde edilen Smarandache eğrilerin grafikleri çizildi ve bazı ilgili sonuçlar verildi.

Anahtar Kelimeler: Minkowski uzayı, spacelike anti-Salkowski eğri, Smarandache eğri, Frenet çatı.

\section{Introduction}

E. Salkowski (1844-1923) defined a family of curves with constant torsion but non-constant curvature with an explicit parametrization [Salkowski,1909]. These curves are known as anti-Salkowski curves in literature. The equation of anti-Salkowski curve was given by Monterde and he showed that the principal normal vector of this curve makes a constant angle with a constant direction [Monterde, 2009]. Turgut and Yilmaz described the Smarandache curves in Minkowski space [Turgut and Yilmaz, 2008; Turgut and Y1lmaz, 2008]. Later, according to the Darboux frame, Bishop frame and Sabban frame, some features of the Smarandache curves were investigated by [Şenyut and 
Sivas, 2013; Bektaş and Yüce 2013; Çetin, Tuncer and Karacan, 2014; Taşköprü and Tosun, 2014; Ali, 2010; Çalışkan and Şenyurt 2015]. Timelike anti-Salkowski curve, spacelike anti-Salkowski curve with a spacelike principal normal and spacelike antiSalkowski curve with a timelike principal normal was introduced by Ali Ahmet [Ali, 2010; Ali, 2009; Ali, 2011]. Şenyurt and Eren also studied the Smarandache curves obtained from the Frenet vectors of the timelike antiSalkowski curve [Şenyurt and Eren, 2019; Şenyurt and Eren, 2019].

In this study, $T N, T B, N B$ and $T N B-$ Smarandache curves, are drawn by a unit vector which is obtained from the linear combination of $T$ the unit tangent vector, $N$ unit principal normal vector and $B$ unit binormal vectors of the spacelike antiSalkowski curve with a timelike principal normal, are defined. The Frenet apparatus of each curve are calculated and the graph of Smarandache curves is given.

\section{Preliminaries}

The Minkowski 3-space $R_{1}^{3}$ is 3-dimensional affine space equipped with a Lorentzian inner product given by

$$
\langle,\rangle=d x_{1}^{2}+d x_{2}^{2}-d x_{3}^{2}
$$

where, $X=\left(x_{1}, x_{2}, x_{3}\right) \in R^{3}$. The vector product of any vectors $X=\left(x_{1}, x_{2}, x_{3}\right)$ and $Y=\left(y_{1}, y_{2}, y_{3}\right)$ in $R_{1}^{3}$ is defined by

$$
X \mathrm{x} Y=-\left|\begin{array}{ccc}
i & j & -k \\
x_{1} & x_{2} & x_{3} \\
y_{1} & y_{2} & y_{3}
\end{array}\right| .
$$

For an arbitrary vector $X \in R_{1}^{3}$, if $\langle X, X\rangle>0$ or $X=0$ then $X$ is a spacelike vector, if $\langle X, X\rangle<0$, then $X$ is a timelike vector, or if $\langle X, X\rangle=0, X \neq 0$, then $X$ is a lightlike ( null) vector. The norm of an arbitrary vector $X \in R_{1}^{3}$ is $\|X\|=\sqrt{|\langle X, X\rangle|}$.

If the tangent vector $\gamma^{\prime}(t)$ of the curve $\gamma: I \rightarrow R_{1}^{3}$ is timelike vector and spacelike vector then $\gamma(t)$ is a timelike curve and spacelike curve, respectively. Let $\gamma(t)$ be regular a spacelike curve. The Frenet vectors, the curvatures and the Frenet formula of the spacelike curve $\gamma(t)$ with a timelike principal normal are

$$
\begin{gathered}
T(t)=\frac{\gamma^{\prime}(t)}{\left\|\gamma^{\prime}(t)\right\|}, \\
B(t)=\frac{\gamma^{\prime}(t) \wedge \gamma^{\prime \prime}(t)}{\left\|\gamma^{\prime}(t) \wedge \gamma^{\prime \prime}(t)\right\|}, \\
N(t)=B(t) \wedge T(t), \\
\kappa(t)=\frac{\left\|\gamma^{\prime}(t) \wedge \gamma^{\prime \prime}(t)\right\|}{\left\|\gamma^{\prime}(t)\right\|^{3}}, \\
\tau(t)=\frac{\left\langle\gamma^{\prime}(t) \wedge \gamma^{\prime \prime}(t), \gamma^{\prime \prime \prime}(t)\right\rangle}{\left\|\gamma^{\prime}(t) \wedge \gamma^{\prime \prime}(t)\right\|^{2}} \\
T^{\prime}=\kappa N, \quad N^{\prime}=\kappa T+\tau B, B^{\prime}=\tau N \\
\text { where } T \text { and } B \text { are spacelike vectors and } N \\
\text { is a timelike vector }\left[\mathrm{O}^{\prime}\right. \text { Neill,1983]. } \\
\text { Definition 2.2. For } m>1 \text { or } m<-1 \text { and } \\
m \in R, \text { let us define the space curve }
\end{gathered}
$$




$$
\gamma_{m}(t)=\frac{n}{4 m}\left(\begin{array}{l}
2 n \cos (t)-\frac{1-n}{1+2 n} \cos ((1+2 n) t) \\
+\frac{1+n}{1-2 n} \cos ((1-2 n) t), \\
2 n \sin (t)-\frac{1-n}{1+2 n} \sin ((1+2 n) t) \\
+\frac{1+n}{1-2 n} \sin ((1-2 n) t), \\
-\frac{1}{m}(2 n t+\sin (2 n t))
\end{array}\right),
$$

where $n=\frac{m}{\sqrt{m^{2}-1}}$. This curve is called the spacelike anti-Salkowski curve with a timelike principal normal (Figure 2.1). The arc length of the spacelike anti-Salkowski curve with a timelike principal normal is $s=\frac{\sinh (n t)}{m}$. The curvature and torsion of this curve $\quad$ are $\quad \kappa(t)=\tan (n t) \quad$ and $\tau(t)=1$,

respectively. In [Ali, 2011], the Frenet frame of the spacelike anti-Salkowski curve with a timelike principal normal is given as follow:

$$
\begin{aligned}
& T(t)=\left(\begin{array}{l}
-n \sin (t) \cos (n t)+\cos (t) \sin (n t), \\
n \cos (t) \cos (n t)+\sin (t) \sin (n t), \\
-\frac{n}{m} \cos (n t)
\end{array}\right), \\
& N(t)=\left(\begin{array}{l}
-\sqrt{n^{2}-1} \sin (t), \\
\sqrt{n^{2}-1} \cos (t), \\
-n
\end{array}\right), \\
& B(t)=\left(\begin{array}{l}
-\cos (t) \cos (n t)-n \sin (t) \sin (n t), \\
-\sin (t) \cos (n t)+n \cos (t) \sin (n t), \\
-\sqrt{n^{2}-1} \sin (n t)
\end{array}\right) .
\end{aligned}
$$

From here, the equation (2.2) becomes as

$$
T^{\prime}=\kappa N, N^{\prime}=\kappa T+B, B^{\prime}=N .
$$

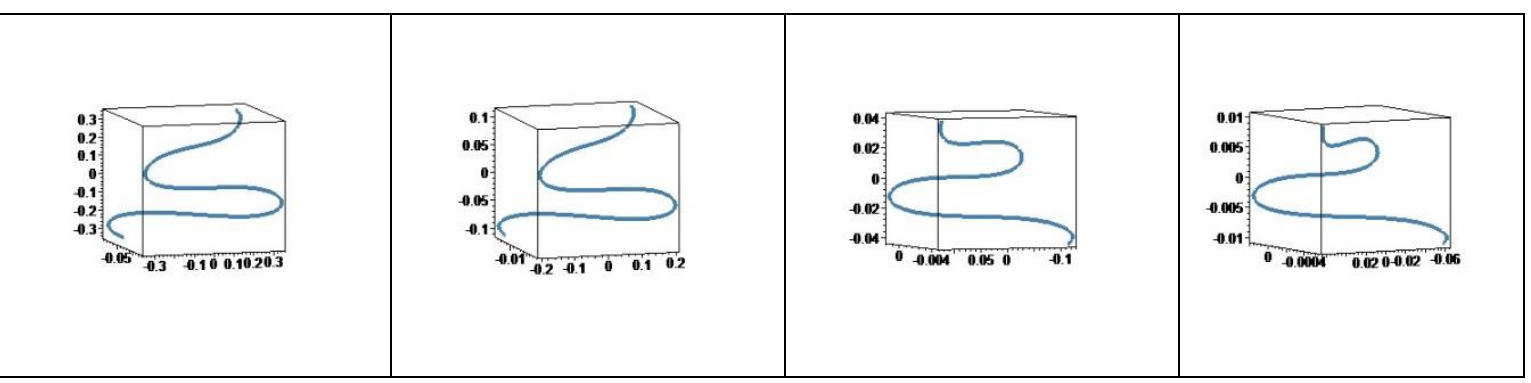

Figure 2.1 $m=\{3,5,8,16\}$ and $t \in[-5,5]$ for spacelike anti-Salkowski curve

3. Smarandache Curves of Spacelike Anti-Salkowski Curve with a Timelike Principal Normal According to Frenet Frame

In this section, we describe Smarandache curves of the spacelike anti-Salkowski curve with a timelike principal normal according to
Frenet frame and we calculate Frenet apparatus of Smarandache curves

Definition 3.1. Let $\gamma_{m}(t)$ be a spacelike antiSalkowski curve with a timelike principal normal. Then $\gamma_{T N}(t)$ Smarandache curve of 
$\gamma_{m}(t)$ can be defined by the frame vectors of and $N$ vectors into the equation $(2.4)$, we get $\gamma_{m}(t)$ such as: $\gamma_{T N}(t)$ curve as follow:

$$
\gamma_{T N}(t)=\frac{1}{\sqrt{2}}(T(t)+N(t))
$$

where $T$ is a spacelike vector and $N$ is a timelike vector (Figure 3.1). Substituting $T$

$$
\gamma_{T N}(t)=\frac{1}{\sqrt{2}}\left(\begin{array}{l}
-n \sin (t) \cos (n t)+\cos (t) \sin (n t) \\
-\sqrt{n^{2}-1} \sin (t), \\
n \cos (t) \cos (n t)+\sin (t) \sin (n t)+ \\
\sqrt{n^{2}-1} \cos (t),-\frac{n}{m} \cos (n t)-n
\end{array}\right)
$$

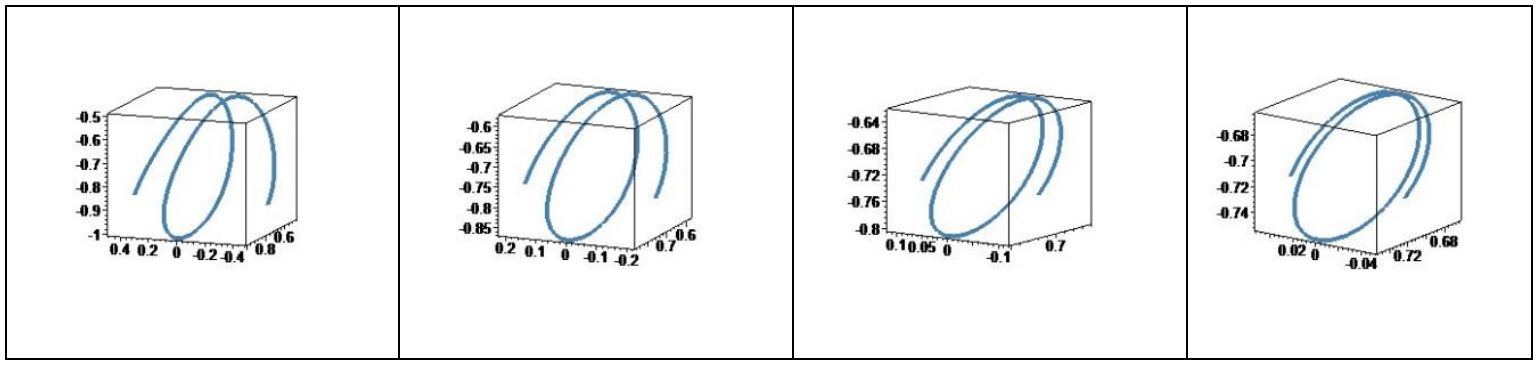

Figure 3.1 $m=\{3,5,8,16\}$ and $t \in[-5,5]$ for $T N-$ Smarandache curve

Theorem 3.1. $\left\{T_{T N}, N_{T N}, B_{T N}\right\}$ Frenet frame of $\gamma_{T N}(t)$ Smarandache curve is given by

$T_{T N}=\kappa T+\kappa N+B$,

$$
\begin{aligned}
N_{T N}= & -\frac{\kappa^{2}+\kappa^{\prime}}{\sqrt{\left|1+2 \kappa^{\prime}+\kappa^{2}\right|}} T-\frac{\kappa^{2}+1+\kappa^{\prime}}{\sqrt{\left|1+2 \kappa^{\prime}+\kappa^{2}\right|}} N \\
& -\frac{\kappa}{\sqrt{\left|1+2 \kappa^{\prime}+\kappa^{2}\right|}} B,
\end{aligned}
$$$$
B_{T N}=\frac{1+\kappa^{\prime}}{\sqrt{\left|1+2 \kappa^{\prime}+\kappa^{2}\right|}} T+\frac{\kappa^{\prime}}{\sqrt{\left|1+2 \kappa^{\prime}+\kappa^{2}\right|}} N
$$$$
-\frac{\kappa}{\sqrt{\left|1+2 \kappa^{\prime}+\kappa^{2}\right|}} B
$$$$
\kappa^{\prime} \neq-\frac{1+\kappa^{2}}{2} \text {. }
$$

Proof: Considering the equation (2.5) in derivate of the equation(3.1), we get

$$
\gamma_{T N}^{\prime}(t)=\frac{1}{\sqrt{2}}(\kappa T+\kappa N+B)
$$

The norm of this equation is found

$$
\left\|\gamma_{T N}^{\prime}(t)\right\|=\frac{1}{\sqrt{2}} .
$$

From the equations (3.3) and(3.4), the tangent vector of $\gamma_{T N}(t)$ is found as

$$
T_{T N}=\kappa T+\kappa N+B
$$

If we take derivate the equation(3.3), we get

$$
\gamma_{T N}^{\prime \prime}(t)=\frac{1}{\sqrt{2}}\left(\left(\kappa^{\prime}+\kappa^{2}\right) T+\left(1+\kappa^{\prime}+\kappa^{2}\right) N+\kappa B\right) .
$$

From the equations (3.3) and(3.6), we find 


$$
\gamma_{T N}^{\prime}(t) \wedge \gamma_{T N}^{\prime \prime}(t)=\frac{1}{2}\left(\left(1+\kappa^{\prime}\right) T+\kappa^{\prime} N-\kappa B\right)
$$

The norm of this equation is

$$
\left\|\gamma_{T N}^{\prime}(t) \wedge \gamma_{T N}^{\prime \prime}(t)\right\|=\frac{1}{2} \sqrt{\left|1+2 \kappa^{\prime}+\kappa^{2}\right|} .
$$

From the equations (3.7) and(3.8), the binormal vector of $\gamma_{T N}(t)$ Smarandache curve is found

$$
\begin{aligned}
B_{T N} & =\frac{1+\kappa^{\prime}}{\sqrt{\left|1+2 \kappa^{\prime}+\kappa^{2}\right|}} T+\frac{\kappa^{\prime}}{\sqrt{1+2 \kappa^{\prime}+\kappa^{2} \mid}} N \\
& -\frac{\kappa}{\sqrt{\left|1+2 \kappa^{\prime}+\kappa^{2}\right|}} B, \\
& \kappa^{\prime} \neq-\frac{1+\kappa^{2}}{2} .
\end{aligned}
$$

From the equations (3.5) and (3.9) the principal normal vector of $\gamma_{T N}(t)$ Smarandache curve is obtained by

$$
\begin{aligned}
& N_{\text {TN }}=-\frac{\kappa^{2}+\kappa^{\prime}}{\sqrt{\left|1+2 \kappa^{\prime}+\kappa^{2}\right|}} T-\frac{\kappa^{2}+1+\kappa^{\prime}}{\sqrt{\left|1+2 \kappa^{\prime}+\kappa^{2}\right|}} N \\
& -\frac{\kappa}{\sqrt{\left|1+2 \kappa^{\prime}+\kappa^{2}\right|}} B, \\
& \kappa^{\prime} \neq-\frac{1+\kappa^{2}}{2} .
\end{aligned}
$$

Theorem 3.2. The curvature and the torsion of $\gamma_{T N}(t)$ Smarandache curve are

$$
\begin{gathered}
\kappa_{T N}=\sqrt{2\left|\left(1+2 \kappa^{\prime}+\kappa^{2}\right)\right|}, \\
\tau_{T N}=\frac{\sqrt{2}\left(\kappa^{\prime \prime}+\kappa \kappa^{\prime}\right)}{1+2 \kappa^{\prime}+\kappa^{2}}, \\
\kappa^{\prime} \neq-\frac{1+\kappa^{2}}{2} 0
\end{gathered}
$$

respectively.

Proof: Considering(2.1), (3.4) and(3.8), we find $\kappa_{T N}$ the curvature of Smarandache curve If we take derivate the equation(3.6), we get

$$
\gamma_{T N}^{\prime \prime \prime}(t)=\frac{1}{\sqrt{2}}\left(\begin{array}{l}
\left(\kappa^{3}+\kappa+3 \kappa \kappa^{\prime}+\kappa^{\prime \prime}\right) T \\
+\left(\kappa^{3}+\kappa+3 \kappa \kappa^{\prime}+\kappa^{\prime \prime}\right) N \\
+\left(\kappa^{2}+1+2 \kappa^{\prime}\right) B
\end{array}\right)
$$

Considering the equations $(3.3),(3.6),(3.8)$ and (3.12) in the equation (2.4), we obtain $\tau_{T N}$ the torsion of the Smarandache curve.

Definition 3.2. Let $\gamma_{m}(t)$ be a spacelike antiSalkowski curve with a timelike principal normal. Then. $\gamma_{T B}(t)$ Smarandache curves of $\gamma_{m}(t)$ can be defined by the frame vectors of $\gamma_{m}(t)$ such as:

$$
\gamma_{T B}(t)=\frac{1}{\sqrt{2}}(T(t)+B(t))
$$

where $T$ is a spacelike vector $B$ is a spacelike vector (Figure 3.2). Substituting $T$ and $B$ vectors into (2.4) the equation, we get $\gamma_{T B}(t)$ curve as follows: 


$$
\gamma_{T B}(t)=\frac{1}{\sqrt{2}}\left(\begin{array}{c}
-n \sin (t) \cos (n t)+\cos (t) \sin (n t) \\
-\cos (t) \cos (n t)-n \sin (t) \sin (n t), \\
n \cos (t) \cos (n t)+\sin (t) \sin (n t) \\
-\sin (t) \cos (n t)+n \cos (t) \sin (n t), \\
-\frac{n}{m} \cos (n t)-\sqrt{n^{2}-1} \sin (n t)
\end{array}\right)
$$

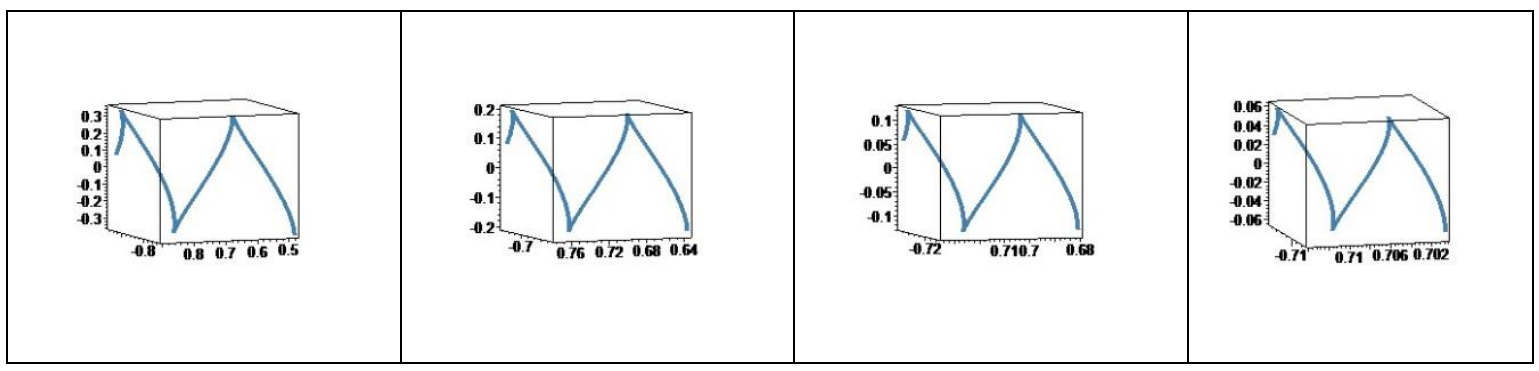

Figure 3.2 $m=\{3,5,8,16\}$ and $t \in[-5,5]$ for $T B-$ Smarandache curve.

Theorem 3.3. $\left\{T_{T B}, N_{T B}, B_{T B}\right\}$ Frenet frame of $\gamma_{T B}(t)$ Smarandache curve is given by $T_{T B}=N$,

$N_{T B}=\frac{\kappa}{\sqrt{1+\kappa^{2}}} T+\frac{1}{\sqrt{1+\kappa^{2}}} B$,

$B_{T B}=-\frac{1}{\sqrt{1+\kappa^{2}}} T+\frac{\kappa}{\sqrt{1+\kappa^{2}}} B$.

Proof: Considering (2.5) in derivate of the equation(3.13), we get

$$
\gamma_{T B}^{\prime}(t)=\frac{1}{\sqrt{2}}(\kappa+1) N
$$

The norm of this equation is

$$
\left\|\gamma_{T B}^{\prime}(t)\right\|=\frac{1}{\sqrt{2}}|\kappa+1| .
$$

From the equations (3.15) and(3.16), the tangent vector of $\gamma_{T B}(t)$ Smarandache curve is found

$$
T_{T N}=N
$$

If we take derivate the equation(3.15), we get

$$
\gamma_{T B}^{\prime \prime}(t)=\frac{1}{\sqrt{2}}\left(\left(\kappa^{2}+\kappa\right) T+\kappa^{\prime} N+(\kappa+1) B\right)
$$

From the equations (3.15) and(3.18), we have

$$
\gamma_{T N}^{\prime}(t) \wedge \gamma_{T N}^{\prime \prime}(t)=\frac{1}{2}\left(-(\kappa+1)^{2} T+\kappa(\kappa+1)^{2} B\right) .
$$

The norm of this equation is

$$
\left\|\gamma_{T N}^{\prime}(t) \wedge \gamma_{T N}^{\prime \prime}(t)\right\|=\frac{1}{2}(\kappa+1)^{2} \sqrt{\kappa^{2}+1}
$$

From the equations (3.19) and(3.20), the binormal vector of $\gamma_{T B}(t)$ Smarandache curve is found 


$$
B_{T B}=-\frac{1}{\sqrt{1+\kappa^{2}}} T+\frac{\kappa}{\sqrt{1+\kappa^{2}}} B
$$

and from the equations $(3.17)$ and (3.21), the principal normal vector of the curve $\gamma_{T B}(t)$ is obtained by

$$
N_{T B}=\frac{\kappa}{\sqrt{1+\kappa^{2}}} T+\frac{1}{\sqrt{1+\kappa^{2}}} B
$$

Theorem 3.4. The curvature and the torsion of $\gamma_{T B}(t)$ Smarandache curve are

$$
\begin{gathered}
\kappa_{T B}=\frac{\sqrt{2} \sqrt{1+\kappa^{2}}}{|\kappa+1|}, \\
\tau_{T B}=-\frac{\sqrt{2} \kappa^{\prime}}{(\kappa+1)\left(1+\kappa^{2}\right)},
\end{gathered}
$$

respectively, where $\kappa \neq-1$.

Proof: Considering the equations (3.16) and (3.20) in the equation(2.1), the curvature $\kappa_{T N}$ of the Smarandache curve is

$$
\kappa_{T B}=\frac{\sqrt{2} \sqrt{1+\kappa^{2}}}{|\kappa+1|}, \kappa \neq-1 .
$$

If we take derivate the equation(3.18), we found

$$
\gamma_{T B}^{\prime \prime \prime}(t)=\frac{1}{\sqrt{2}}\left(\begin{array}{l}
\left(3 \kappa \kappa^{\prime}+\kappa^{\prime}\right) T \\
+\left(\kappa^{3}+\kappa^{2}+\kappa+1+\kappa^{\prime \prime}\right) N \\
+2 \kappa^{\prime} B
\end{array}\right)
$$

From the equations $(3.15),(3.18),(3.20)$ and (3.24), $\gamma_{T B}(t)$ the torsion of the Smarandache curve is obtained by

$$
\tau_{T B}=-\frac{\sqrt{2} \kappa^{\prime}}{(\kappa+1)\left(1+\kappa^{2}\right)}, \kappa \neq-1
$$

Definition 3.3. Let $\gamma_{m}(t)$ be a spacelike antiSalkowski curve with a timelike principal normal. Then. $\gamma_{N B}(t)$ Smarandache curves of $\gamma_{m}(t)$ can be defined by the frame vectors of $\gamma_{m}(t)$ such as:

$$
\gamma_{N B}(t)=\frac{1}{\sqrt{2}}(N(t)+B(t))
$$

where, $N$ is timelike vector and $B$ is a spacelike vector (Figure 3.3). Substituting $N$ and $B$ vectors into (2.4) the equation, we get $\gamma_{N B}(t)$ curve as follows:

$$
\gamma_{N B}(t)=\frac{1}{\sqrt{2}}\left(\begin{array}{l}
-\cos (t) \cos (n t)-n \sin (t) \sin (n t) \\
-\sqrt{n^{2}-1} \sin (t) \\
-\sin (t) \cos (n t)+n \cos (t) \sin (n t) \\
+\sqrt{n^{2}-1} \cos (t) \\
-\sqrt{n^{2}-1} \sin (n t)-n
\end{array}\right)
$$




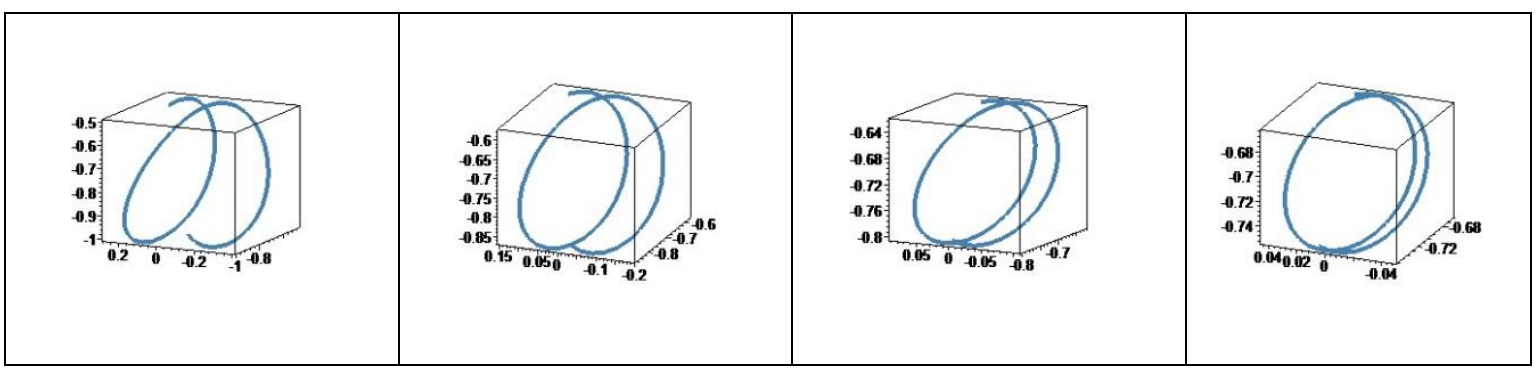

Figure 3.3 $m=\{3,5,8,16\}$ and $t \in[-5,5]$ for $N B-$ Smarandache curve.

Theorem 3.5. $\left\{T_{N B}, N_{N B}, B_{N B}\right\}$ Frenet frame The norm of this equation is found of $\gamma_{N B}(t)$ Smarandache curve is given by

$T_{N B}=\frac{\kappa}{|\kappa|} T+\frac{1}{|\kappa|} N+\frac{1}{|\kappa|} B$

$$
\begin{aligned}
N_{N B} & =-\frac{\kappa^{3}}{|\kappa| \sqrt{\left|\kappa^{6}+\kappa^{4}-2 \kappa^{\prime} \kappa^{3}\right|}} T \\
& +\frac{\kappa^{\prime}-\kappa^{3}-\kappa^{2}}{|\kappa| \sqrt{\left|\kappa^{6}+\kappa^{4}-2 \kappa^{\prime} \kappa^{3}\right|}} N \\
& +\frac{-\kappa^{2}+\kappa \kappa^{\prime}}{|\kappa| \sqrt{\left|\kappa^{6}+\kappa^{4}-2 \kappa^{\prime} \kappa^{3}\right|}} B,
\end{aligned}
$$

$$
\begin{aligned}
& B_{N B}=\frac{\kappa^{2}}{\sqrt{\left|\kappa^{6}+\kappa^{4}-2 \kappa^{\prime} \kappa^{3}\right|}} T \\
&+\frac{\kappa^{\prime}}{\sqrt{\left|\kappa^{6}+\kappa^{4}-2 \kappa^{\prime} \kappa^{3}\right|}} N \\
&+\frac{\kappa^{\prime}-\kappa^{3}}{\sqrt{\left|\kappa^{6}+\kappa^{4}-2 \kappa^{\prime} \kappa^{3}\right|}} B, \\
& \kappa^{\prime} \neq \frac{\kappa\left(1+\kappa^{2}\right)}{2} \text { for } \kappa \neq 0 .
\end{aligned}
$$

Proof: If we take derivate the equation, (3.25)

we get

$$
\gamma_{N B}^{\prime}(t)=\frac{1}{\sqrt{2}}(\kappa T+N+B) .
$$

$$
\left\|\gamma_{N B}^{\prime}(t)\right\|=\frac{1}{\sqrt{2}}|\kappa| .
$$

From the equations (3.27) and(3.28), the tangent vector of $\gamma_{N B}(t)$ Smarandache curve is

$$
T_{N B}=\frac{\kappa}{|\kappa|} T+\frac{1}{|\kappa|} N+\frac{1}{|\kappa|} B, \kappa \neq 0 . .
$$

If we take derivate the equation (3.27), we have

$\gamma_{N B}^{\prime \prime}(t)=\frac{1}{\sqrt{2}}\left(\left(\kappa^{\prime}+\kappa\right) T+\left(1+\kappa^{2}\right) N+B\right)$.

From the equations (3.27) and (3.30), we found

$$
\gamma_{N B}^{\prime}(t) \wedge \gamma_{N B}^{\prime \prime}(t)=\frac{1}{2}\left(\kappa^{2} T+\kappa^{\prime} N+\left(\kappa^{\prime}-\kappa^{3}\right) B\right) .
$$

The norm of the equation (3.31) is

$$
\left\|\gamma_{N B}^{\prime}(t) \wedge \gamma_{N B}^{\prime \prime}(t)\right\|=\frac{1}{2} \sqrt{\left|\kappa^{6}+\kappa^{4}-2 \kappa^{\prime} \kappa^{3}\right|}
$$

From the equations (3.31) and(3.32), the 
binormal vector of $\gamma_{N B}(t)$ Smarandache curve is found by

$$
\begin{gathered}
\kappa_{N B}=\frac{\sqrt{2} \sqrt{\left|\kappa^{6}+\kappa^{4}-2 \kappa^{\prime} \kappa^{3}\right|}}{|\kappa|^{3}}, \\
\tau_{N B}=\frac{\sqrt{2}\left(\kappa^{2} \kappa^{\prime \prime}+\kappa^{2} \kappa^{\prime}-3 \kappa \kappa^{\prime 2}\right)}{\left|\kappa^{6}+\kappa^{4}-2 \kappa^{\prime} \kappa^{3}\right|}, \\
\kappa^{\prime} \neq \frac{\kappa\left(1+\kappa^{2}\right)}{2} \text { for } \kappa \neq 0,
\end{gathered}
$$

receptively.

$$
\begin{gathered}
+\frac{\kappa^{\prime}-\kappa^{3}}{\sqrt{\left|\kappa^{6}+\kappa^{4}-2 \kappa^{\prime} \kappa^{3}\right|}} B, \\
\kappa^{\prime} \neq \frac{\kappa\left(1+\kappa^{2}\right)}{2} \text { for } \kappa \neq 0 .
\end{gathered}
$$

Proof: From the equations $(2.1),(3.28)$ and (3.32), we find the curvature of the curve $\gamma_{N B}(t)$.

And from the equations (3.29) and (3.33), the principal normal vector of the curve

$$
\begin{aligned}
\gamma_{N B}(t) & \text { is obtained } \\
N_{N B} & =-\frac{\kappa^{3}}{|\kappa| \sqrt{\left|\kappa^{6}+\kappa^{4}-2 \kappa^{\prime} \kappa^{3}\right|}} T \\
& +\frac{\kappa^{\prime}-\kappa^{3}-\kappa^{2}}{|\kappa| \sqrt{\left|\kappa^{6}+\kappa^{4}-2 \kappa^{\prime} \kappa^{3}\right|}} N \\
& +\frac{-\kappa^{2}+\kappa \kappa^{\prime}}{|\kappa| \sqrt{\left|\kappa^{6}+\kappa^{4}-2 \kappa^{\prime} \kappa^{3}\right|}} B, \\
& \kappa^{\prime} \neq \frac{\kappa\left(1+\kappa^{2}\right)}{2} \text { for } \kappa \neq 0 .
\end{aligned}
$$

Theorem 3.6. The curvature and the torsion of $\gamma_{N B}(t)$ Smarandache curve are

If we take derivate the equation(3.30), we get

$$
\gamma_{T N}^{\prime \prime \prime}(t)=\frac{1}{\sqrt{2}}\left(\begin{array}{l}
\left(\kappa^{3}+\kappa+\kappa^{\prime \prime}+\kappa^{\prime}\right) T \\
+\left(\kappa^{2}+1+3 \kappa \kappa^{\prime}\right) N \\
+\left(1+\kappa^{2}\right) B
\end{array}\right)
$$

From the equations (3.27), (3.30), (3.32) and (3.36), the torsion of the curve $\gamma_{N B}(t)$ is obtained

Definition 3.4. Let $\gamma_{m}(t)$ be a spacelike antiSalkowski curve with a timelike principal normal. Then. $\gamma_{T N B}(t)$ Smarandache curves of $\gamma_{m}(t)$ can be defined by the frame vectors of $\gamma_{m}(t)$ such as:

$$
\gamma_{T N B}(t)=\frac{1}{\sqrt{3}}(T(t)+N(t)+B(t))
$$

where $T$ is a spacelike vector, $N$ is a timelike vector and $B$ is a spacelike vector (Figure 
3.4). Substituting $T, N$ and $B$ vectors into (2.4) the equation, we get $\gamma_{T N B}(t)$ curve as follows:

$$
\gamma_{T N B}(t)=\frac{1}{\sqrt{3}}\left(\begin{array}{l}
-n \sin (t) \cos (n t)+\cos (t) \sin (n t) \\
-\cos (t) \cos (n t)-n \sin (t) \sin (n t) \\
-\sqrt{n^{2}-1} \sin (t), \\
n \cos (t) \cos (n t)+\sin (t) \sin (n t) \\
-\sin (t) \cos (n t)+n \cos (t) \sin (n t) \\
+\sqrt{n^{2}-1} \cos (t), \\
-\frac{n}{m} \cos (n t)-\sqrt{n^{2}-1} \sin (n t)-n
\end{array}\right)
$$

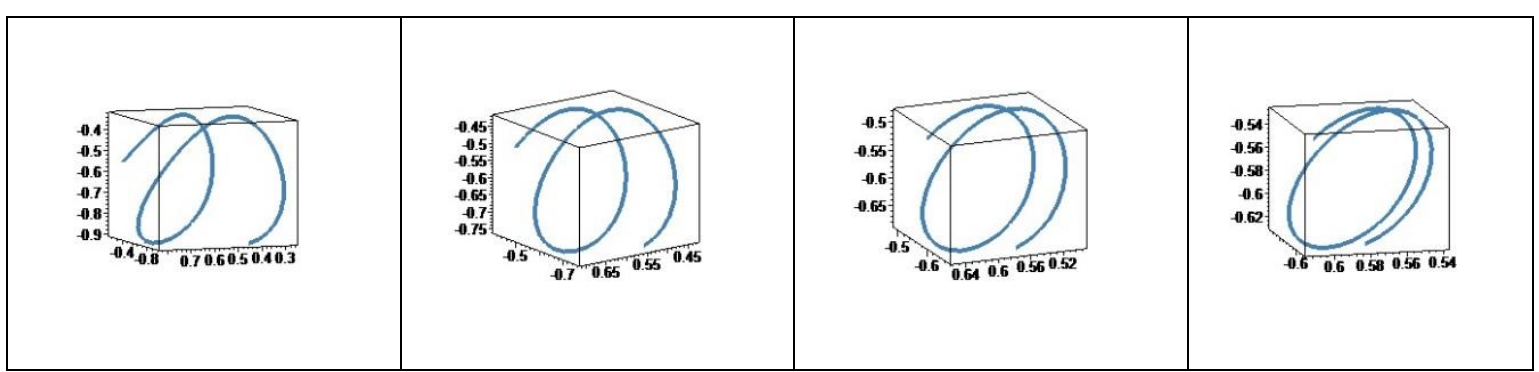

Figure 3.4 $m=\{3,5,8,16\}$ and $t \in[-5,5]$ for $T N B-$ Smarandache curve.

Theorem 3.7. $\left\{T_{T N B}, N_{T N B}, B_{T N B}\right\}$ Frenet frame of $\gamma_{T N B}(t)$ Smarandache curve is given by

$$
\begin{aligned}
T_{T N B} & =\frac{\kappa}{\sqrt{|-2 \kappa|}} T+\frac{\kappa+1}{\sqrt{|-2 \kappa|}} N+\frac{1}{\sqrt{|-2 \kappa|}} B \\
N_{T N B} & =\frac{2 \kappa^{3}+2 \kappa^{2}+\kappa \kappa^{\prime}}{\sqrt{|-2 \kappa|\left|\left(\kappa^{\prime}-2 \kappa\right)^{2}-\kappa^{\prime 2}+\left(2 \kappa^{2}+\kappa^{\prime}\right)^{2}\right|}} T \\
& +\frac{2 \kappa^{3}+2 \kappa+\kappa \kappa^{\prime}-\kappa^{\prime}}{\sqrt{|-2 \kappa|\left|\left(\kappa^{\prime}-2 \kappa\right)^{2}-\kappa^{\prime 2}+\left(2 \kappa^{2}+\kappa^{\prime}\right)^{2}\right|}} N \\
& +\frac{2 \kappa^{2}+2 \kappa-\kappa^{\prime}}{\sqrt{|-2 \kappa|\left|\left(\kappa^{\prime}-2 \kappa\right)^{2}-\kappa^{\prime 2}+\left(2 \kappa^{2}+\kappa^{\prime}\right)^{2}\right|}} B
\end{aligned}
$$

$$
\begin{gathered}
B_{T N B}=\frac{\kappa^{\prime}-2 \kappa}{\sqrt{\left|\left(\kappa^{\prime}-2 \kappa\right)^{2}-\kappa^{\prime 2}+\left(2 \kappa^{2}+\kappa^{\prime}\right)^{2}\right|}} T \\
+\frac{\kappa^{\prime}}{\sqrt{\left|\left(\kappa^{\prime}-2 \kappa\right)^{2}-\kappa^{\prime 2}+\left(2 \kappa^{2}+\kappa^{\prime}\right)^{2}\right|}} N \\
+\frac{2 \kappa^{2}+\kappa^{\prime}}{\sqrt{\left|\left(\kappa^{\prime}-2 \kappa\right)^{2}-\kappa^{\prime 2}+\left(2 \kappa^{2}+\kappa^{\prime}\right)^{2}\right|}} B, \\
\left(\kappa^{\prime}-2 \kappa\right)^{2}-\kappa^{\prime 2}+\left(2 \kappa^{2}+\kappa^{\prime}\right)^{2} \neq 0, \kappa \neq 0 .
\end{gathered}
$$

Proof: If we take derivate the equation(3.37), we get

$$
\gamma_{T N B}^{\prime}(t)=\frac{1}{\sqrt{3}}(\kappa T+(\kappa+1) N+B) .
$$

The norm of this equation is

$$
\left\|\gamma_{T N}^{\prime}(t)\right\|=\frac{1}{\sqrt{3}} \sqrt{|-2 \kappa|} .
$$


From the equations (3.39) and(3.40), the From the equations (3.41) and(3.45), the tangent vector of $\gamma_{T N B}(t)$ Smarandache curve principal normal of the curve $\gamma_{T N B}(t)$ is is found obtained by

$$
T_{T N B}=\frac{\kappa}{\sqrt{|-2 \kappa|}} T+\frac{\kappa+1}{\sqrt{|-2 \kappa|}} N+\frac{1}{\sqrt{|-2 \kappa|}} B, \kappa \neq 0 .
$$

The derivate of the equation (3.39) is

$$
\gamma_{T N}^{\prime \prime}(t)=\frac{1}{\sqrt{3}}\left(\begin{array}{l}
\left(\kappa^{\prime}+\kappa+\kappa^{2}\right) T \\
+\left(\kappa^{\prime}+\kappa^{2}+1\right) N+(\kappa+1) B
\end{array}\right) .
$$

From the equations (3.39) and(3.42), it is found

$$
\gamma_{T N B}^{\prime}(t) \wedge \gamma_{T N B}^{\prime \prime}(t)=\frac{1}{3}\left(\begin{array}{l}
\left(\kappa^{\prime}-2 \kappa\right) T \\
+\kappa^{\prime} N+\left(2 \kappa^{2}+\kappa^{\prime}\right) B
\end{array}\right)(
$$

The norm of this equation (3.43) is

$$
\left\|\gamma_{T N B}^{\prime}(t) \wedge \gamma_{T N B}^{\prime \prime}(t)\right\|=\frac{1}{3} \sqrt{\left(\kappa^{\prime}-2 \kappa\right)^{2}-\kappa^{\prime 2}+\left(2 \kappa^{2}+\kappa^{\prime}\right)^{2}}
$$

From the equations (3.43) and(3.44), the binormal vector of the curve $\gamma_{T N B}(t)$ is found by

$$
\begin{gathered}
B_{\pi N B}=\frac{\kappa^{\prime}-2 \kappa}{\sqrt{\left|\left(\kappa^{\prime}-2 \kappa\right)^{2}-\kappa^{\prime 2}+\left(2 \kappa^{2}+\kappa^{\prime}\right)^{2}\right|}} T \\
+\frac{\kappa^{\prime}}{\sqrt{\left|\left(\kappa^{\prime}-2 \kappa\right)^{2}-\kappa^{\prime 2}+\left(2 \kappa^{2}+\kappa^{\prime}\right)^{2}\right|}} N \\
+\frac{2 \kappa^{2}+\kappa^{\prime}}{\sqrt{\left|\left(\kappa^{\prime}-2 \kappa\right)^{2}-\kappa^{\prime 2}+\left(2 \kappa^{2}+\kappa^{\prime}\right)^{2}\right|}} B, \\
\left(\kappa^{\prime}-2 \kappa\right)^{2}-\kappa^{\prime 2}+\left(2 \kappa^{2}+\kappa^{\prime}\right)^{2} \neq 0 .
\end{gathered}
$$

$$
\begin{gathered}
N_{n a s}=\frac{2 \kappa^{3}+2 \kappa^{2}+\kappa \kappa^{\prime}}{\sqrt{|-2 \kappa|\left|\left(\kappa^{\prime}-2 \kappa\right)^{2}-\kappa^{\prime 2}+\left(2 \kappa^{2}+\kappa^{\prime}\right)^{2}\right|}} T \\
+\frac{2 \kappa^{3}+2 \kappa+\kappa \kappa^{\prime}-\kappa^{\prime}}{\sqrt{|-2 \kappa|\left|\left(\kappa^{\prime}-2 \kappa\right)^{2}-\kappa^{\prime 2}+\left(2 \kappa^{2}+\kappa^{\prime}\right)^{2}\right|}} N \\
+\frac{2 \kappa^{2}+2 \kappa-\kappa^{\prime}}{\sqrt{|-2 \kappa|\left|\left(\kappa^{\prime}-2 \kappa\right)^{2}-\kappa^{\prime 2}+\left(2 \kappa^{2}+\kappa^{\prime}\right)^{2}\right|}} B \\
\left(\kappa^{\prime}-2 \kappa\right)^{2}-\kappa^{\prime 2}+\left(2 \kappa^{2}+\kappa^{\prime}\right)^{2} \neq 0, \kappa \neq 0 .
\end{gathered}
$$

Theorem 3.8. The curvature and the torsion of $\gamma_{T N B}(t)$ Smarandache curve are

$$
\begin{gathered}
\kappa_{T N B}=\frac{\sqrt{3} \sqrt{\left(\kappa^{\prime}-2 \kappa\right)^{2}-\kappa^{\prime 2}+\left(2 \kappa^{2}+\kappa^{\prime}\right)^{2} \mid}}{|-2 \kappa| \sqrt{|-2 \kappa|}}, \\
\tau_{T N B}=\frac{\sqrt{3}\left(3 \kappa^{\prime 2}-2 \kappa^{2} \kappa^{\prime}-2 \kappa^{\prime} \kappa-2 \kappa \kappa^{\prime \prime}\right)}{\left|\left(\kappa^{\prime}-2 \kappa\right)^{2}-\kappa^{\prime 2}+\left(2 \kappa^{2}+\kappa^{\prime}\right)^{2}\right|}, \\
\left(\kappa^{\prime}-2 \kappa\right)^{2}-\kappa^{\prime 2}+\left(2 \kappa^{2}+\kappa^{\prime}\right)^{2} \neq 0, \kappa \neq 0,
\end{gathered}
$$

respectively.

Proof: From the equations $(2.1),(3.40)$ and (3.44), we find the curvature of $\gamma_{T N B}(t)$ Smarandache curve. The derivate of the equation (3.42) is

$$
\gamma_{T N}^{\prime \prime \prime}(t)=\frac{1}{\sqrt{3}}\left(\begin{array}{l}
\left(\kappa^{3}+\kappa+3 \kappa \kappa^{\prime}+\kappa^{\prime}+\kappa^{\prime \prime}\right) T \\
+\left(\kappa^{3}+\kappa^{2}+\kappa+3 \kappa \kappa^{\prime}+1+\kappa^{\prime \prime}\right) N \\
+\left(\kappa^{2}+1+2 \kappa^{\prime}\right) B .
\end{array}\right)
$$


From the equations $(3.39),(3.42),(3.44)$ and (3.48), the torsion of the curve $\gamma_{T N B}(t)$ is obtained

Corollary 1. $T N$ and $N B-$ Smarandache curves of spacelike anti-Salkowski with a timelike principal normal are spacelike curves with a timelike principal normal.

Proof: From theorem 3.1 and theorem 3.1, the proof is easily seen.

Corollary 2. $T B$ and $T N B-$ Smarandache curves of spacelike anti-Salkowski with a timelike principal normal are timelike curves.

Proof: From theorem 3.3 and theorem 3.7, the proof is easily seen.

Corollary 3. $T B-$ Smarandache curve is the evolute of the spacelike anti-Salkowski curve with a timelike principal normal.

Proof: From the equations (2.4) and (3.17), we get

$$
\left\langle T, T_{T B}\right\rangle=\langle T, N\rangle=0 .
$$

In that case, we call that $T B-$ Smarandache curve is the evolute of the spacelike antiSalkowski curve with a timelike principal normal

\section{References}

Ali, A.T. (2010) Special Smarandache Curves in the Euclidian Space, International Journal of Mathematical Combinatorics, 2,30-36.
Ali, A.T. (2010). Timelike Salkowski and anti-Salkowski curves in Minkowski 3- space. J. Adv. Res. Dyn. Cont. Syst., 2, 17-26.

Ali, A.T. (2009). Spacelike Salkowski and anti-Salkowski curves with spacelike principal normal in Minkowski 3-space. Int. J. Open Problems Comp. Math. 2 451-460.

Ali, A.T. (2011). Spacelike Salkowski and anti-Salkowski curves with timelike principal normal in Minkowski 3-space. Mathematica Aeterna, Vol.1, No.04, 201-210.

Bektaş, Ö. and Yüce, S. (2013) Special Smarandache Curves According to Darboux Frame in Euclidean 3-Space, Romanian Journal of Mathematics and Computer Science, 3(1), 48-59.

Çalışkan, A. and Şenyurt, S. (2015). Smarandache Curves in Terms of Sabban Frame of Spherical Indicatrix Curves, Gen. Math. Notes, 31(2),1-15.

Çetin, M. Tuncer, Y. and Karacan, M.K. (2014) Smarandache Curves According to Bishop Frame in Euclidean 3-Space, Gen. Math. Notes, 20, 50-66.

Monterde, J. (2009). Salkowski curves revisited: A family of curves with constant curvature and non-constant torsion, Computer Aided Geometric Design, 26(3), 271- 278.

O'Neill, B. (1983). Semi-Riemannian Differential Geometry, Academic Press, USA.

Salkowski, E. (1909). Zur Transformation von Raumkurven, Math. Ann., 66, 517-557.

Şenyurt, S. and Sivas, S. (2013). An Application of Smarandache Curve, University of Ordu Journal of Science and Technology, 3(1), 46-60.

Şenyurt, S. and Eren, K. (2019). Smarandache curves of timelike anti-Salkowski curve according to Frenet frame, Blacksea 1. 
International Multidisciplinary Scientific Sociedade Paranaense de Matematica 3 Works Congress, 667-679. Srie.,32(1), 51-59.

Şenyurt, S. and Eren, K. (2019). Smarandache Turgut, M. and Yilmaz, S. (2008). curves of timelike Salkowski curve according Smarandache Curves in Minkowski to Frenet frame, Blacksea 1. International Spacetime, International J.Math. Combin., 3, Multidisciplinary Scientific Works Congress, 51-55.

680-692.

Turgut, M. and Yilmaz, S. (2008) On the Taşköprü, K. and Tosun, M. (2014) Differential Geometry of the curves in Smarandache Curves on $S^{2}$, Boletim da Minkowski spacetime I, Int. J. Contemp. Math. Sci. 3(27), 1343-1349. 